\title{
Modulation Index Estimation of Frequency and Phase Modulated Signals
}

\author{
Geng Peng ${ }^{1,2}$, Derong Cai ${ }^{1}$, Zhiqiang $\mathrm{He}^{1}$, Zhitao Huang ${ }^{2}$ \\ ${ }^{1}$ Naval Academy of Armament, Beijing, China \\ ${ }^{2}$ School of Electronic Science and Engineering, National University of Defense Technology, Hunan, China \\ E-mail:Andrew.higgins@csiro.au,Leonie.pearson@csiro.au,Luis.laredo@csiro.au \\ Received July 2, 2010; revised August 5, 2010; accepted September 6, 2010
}

\begin{abstract}
Modulation index estimation is important in the demodulation and recognition of Angle Modulation (AM) signals which include Frequency Modulation (FM) and Phase Modulation (PM) signals. In this paper, we firstly analyzed the AM signals with baseband modulation types, such as monotone, PSK, FSK, in the time and frequency domains. Then, we established a unified mathematical representation for the AM signals. Finally, we derived a blind estimation algorithm for the modulation index without using any prior knowledge. Simulation results verified the capabilities of the proposed algorithm.
\end{abstract}

Keywords: Information Processing Technology, Blind Estimation, Modulation Index, AM Signal

\section{Introduction}

Angle Modulation (AM), a general form of Frequency Modulation (FM) and Phase Modulation (PM), is an important nonlinear analogue modulation mode used in various communication systems. AM signal transmits information by changing (switching) carrier frequencies or phases according to the variation of the baseband signal. The modulation recognition algorithms for the FM and PM signals can be found in [1-7]. Besides, the modulation index ranges considered in these literatures are listed in Table 1.

It can be seen from Table 1 that the current analogue FM/PM modulation recognition algorithms essentially use the differences in modulation indices. Once these indices are effectively estimated, the FM/PM analogue signals can be identified according to its range in the practical application. As a result, the AM signals can be demodulated blindly based on the modulation index and modulation type. Therefore, the blind estimation of the AM index is of great significance.

As far as we know, there are few researches on the modulation index estimation yet. In this paper, we analyze the AM signal with baseband signals, such as monotone, FSK, PSK, in the TF domain, and establish a unified mathematical expression to help propose an AM index estimation method, which requires no prior knowledge of the signals.

The remainder is arranged as follows: the signal model is established in Section 2; in Section 3, the blind estimation method for the modulation index is derived; the simulation experiments and conclusions are given in Section 4 and Section 5, respectively.

\section{Signal Model}

AM signal is usually expressed as follows [8]:

$$
s(t)=A_{c} \cos \left[w_{c} t+\varphi(t)\right]
$$

where $A_{c}$ is a constant carrier amplitude, $w_{c}=2 \pi f c, \varphi(t)$ is the instant phase offset.

For the PM signal, the instant phase offset proportionally changes with the baseband modulation signal, namely:

$$
\begin{gathered}
\varphi(t)=k_{p} m(t) \\
S_{\mathrm{PM}}(t)=A_{c} \cos \left[w_{c} t+k_{p} m(t)\right]
\end{gathered}
$$

Table 1. Modulation index ranges of analogue FM/PM modulation recognition algorithms in literatures.

\begin{tabular}{ccc}
\hline literatures & $\begin{array}{c}\text { phase modulation } \\
\text { index } m_{p} \text { of PM signals }\end{array}$ & $\begin{array}{c}\text { frequency modulation } \\
\text { index } m_{f} \text { of FM signals }\end{array}$ \\
\hline Ref. [1] & {$[-\pi, \pi]$} & $(1.0,2.0,5.0) \times 2 \pi$ \\
Ref. [2] & $\leq \pi / 2$ & $5.0 \times 2 \pi$ \\
Ref. [3-4] & $\leq 1.5$ & $\geq 5.0$ \\
Ref. [5-6] & $\pi /(18,12,9)$ & $(1,5,10) \times 2 \pi$ \\
Ref. [7] & & $m_{p}>>m_{f}$ \\
\hline
\end{tabular}


where $k_{p}$ is a constant phase offset, $m(t)$ is the baseband modulation signal.

For the FM signal, the change rate of the instant phase offset varies with the baseband signal proportionally, namely:

$$
\begin{gathered}
\varphi(t)=2 \pi k_{f} \int_{-\infty}^{t} m(\tau) \mathrm{d} \tau \\
s_{\mathrm{FM}}(t)=A_{c} \cos \left[w_{c} t+2 \pi k_{f} \int_{-\infty}^{t} m(\tau) \mathrm{d} \tau\right]
\end{gathered}
$$

where $k_{f}$ is a constant frequency offset, which is also named as the frequency sensitivity of a frequency modulator.

\section{Blind AM Index Estimation Method}

In this section, we first analyze the AM signals with different baseband modulation types in the TF domain. Then, we establish a unified mathematical expression of these signals and deduce a blind estimation method for the AM index.

\subsection{The TF Analysis of the AM Signals}

The AM signal with monotone baseband is analyzed in the TF domain first. Then, the baseband modulation types, such as PSK, FSK, are also taken into account. At last, the uniform mathematical expression of the AM signal is obtained.

For the monotone baseband denoted as $m(t)=V_{m} \cos (\Omega t)$ $\left(\Omega=2 \pi f_{m}\right)$, the $s(t)$ can be given by

$$
\begin{gathered}
s(t)=\left\{\begin{array}{cc}
A_{c} \cos \left[w_{c} t+m_{p} \cos (\Omega t)\right], & \mathrm{PM} \\
A_{c} \cos \left[w_{c} t+m_{f} \sin (\Omega t)\right], & \mathrm{FM}
\end{array}\right. \\
\left\{\begin{array}{l}
m_{p}=k_{p} \cdot V_{m} \\
m_{f}=k_{f} \cdot V_{m} / f_{m}
\end{array}\right.
\end{gathered}
$$

where $m_{p}$ and $m_{f}$ are the phase modulation index and the frequency modulation index respectively [8].

The PM signal can be rewritten in an exponential form as

$$
s_{\mathrm{PM}}(t)=A_{c} \cdot \operatorname{Re}\left[\mathrm{e}^{\mathrm{j} w_{c} t} \cdot \mathrm{e}^{\mathrm{j} m_{p} \cos \Omega t}\right]
$$

where $\operatorname{Re}[x(t)]$ is the real part of the function $x(t)$, $\mathrm{e}^{\mathrm{j} m_{p} \cos \Omega t}$ is the periodic function of $\Omega$. Then, the Fourier expansion of the PM signal is:

$$
\begin{aligned}
S_{\mathrm{PM}}(t) & =A_{c} \cdot \operatorname{Re}\left[\mathrm{e}^{\mathrm{j} w_{c} t} \cdot \mathrm{e}^{\mathrm{j} m_{p} \sin (\Omega t+\pi / 2)}\right] \\
& =A_{c} \cdot \operatorname{Re}\left\{\sum_{n=-\infty}^{\infty} J_{n}\left(m_{p}\right) \mathrm{e}^{\mathrm{j}\left[\left(w_{c}+n \Omega t\right) t\right]} \mathrm{e}^{\mathrm{j}(n \pi / 2)}\right\}
\end{aligned}
$$

where $J_{n}(x)$ is type I Bessel function of $x$ with an order $n$.

Considering the baseband signal $m(t)=V_{m} \cos (\Omega t)$, FM and PM signals can be expressed in frequency domain as:

$$
\left\{\begin{array}{l}
\mathrm{PM}: \sum_{n=-\infty}^{\infty}\left\{\delta\left[f-f_{0}\right]+\delta\left[f+f_{0}\right]\right\} \cdot J_{n}\left(m_{p}\right) \cos (n \pi / 2) \\
\mathrm{FM}: \sum_{n=-\infty}^{\infty}\left\{\delta\left[f-f_{0}\right]+\delta\left[f+f_{0}\right]\right\} \cdot J_{n}\left(m_{f}\right)
\end{array}\right.
$$

where $f_{0}=f_{c}+n f_{m}$.

Similarly, FM and PM signals with the baseband signal $m(t)=V_{m} \sin (\Omega t)$ can be expressed in frequency domain as:

$$
\left\{\begin{array}{l}
\mathrm{PM}: \sum_{n=-\infty}^{\infty}\left\{\delta\left[f-f_{0}\right]+\delta\left[f+f_{0}\right]\right\} \cdot J_{n}\left(m_{p}\right) \\
\mathrm{FM}: \sum_{n=-\infty}^{\infty}\left\{\delta\left[f-f_{0}\right]+\delta\left[f+f_{0}\right]\right\} \cdot J_{n}\left(m_{f}\right) \cos (n \pi / 2)
\end{array}\right.
$$

It can be seen from (6), (10) and (11) that the FM and PM signals with monotone baseband are essentially identical in the TF domain.

For the PSK baseband case, $m(t)$ is represented as:

$$
m(t)=g(t) \cos \left[2 \pi f_{m} t+2 \pi(i-1) / M\right], 0 \leq t \leq T_{c}
$$

where $g(t)$ is the response function of the shaping pulse, $i=0, \cdots, M-1, T_{c}$ is the time interval of the code chip.

For the FSK baseband case, $m(t)$ is written as:

$$
\begin{aligned}
m(t) & =g(t) \cos \left[\frac{\pi}{T_{c}}\left(n_{c}+i\right) t\right] \\
& =g(t) \cos \left(2 \pi f_{m} t+\frac{i \pi}{T_{c}} t\right), \quad 0 \leq t \leq T_{c}
\end{aligned}
$$

where $f_{m}=n_{c} /\left(2 T_{c}\right)$ and $n_{c}$ is an integer.

Generally, signals such as PSK and FSK take the sinusoidal signals as the carrier. The information is transmitted by varying some of the parameters of the carrier. It can be drawn from (12), (13) that both the PSK and FSK basebands are monotones with amplitude function $g(t)$ in a code period $T_{c}$. (When the function is a rectangular pulse, $g(t)=1)$. To transmit information, the initial phase and/or frequency for different code intervals are different. So, it is representative to analyze the AM signal with monotone baseband in the TF domain. Accordingly, the subsequent investigation takes the monotone baseband modulation signal as an example.

To facilitate the analysis process, a unified mathematical expression of AM signals with baseband modulations such as monotone, PSK, FSK, is given as follows:

$$
s(t)=A_{c} \cos \left[w_{c} t+m_{p} g(t) \cos \left(\Omega t+\theta_{1}\right)+\theta_{0}\right], 0 \leq t \leq T_{c}
$$

where $\theta_{0}$ is the initial phase of the carrier, $\theta_{1}$ is the initial phase of baseband modulation signal and $g(t)$ can be a square pulse. 


\subsection{Derivation of the Blind Modulation Index Estimation Method}

The monotone baseband modulation FM signal shown in (5) is considered here as an example. After amplitude normalization and differentiating, Equation (5) evolves to:

$$
\begin{aligned}
\frac{\mathrm{d} s_{\mathrm{FM}}(t)}{\mathrm{d} t} & =\frac{\mathrm{d} A}{\mathrm{~d} t} \cos \left[w_{c} t+2 \pi k_{f} \int_{-\infty}^{t} m(\tau) \mathrm{d} \tau\right] \\
& -A\left[w_{c}+2 \pi k_{f} m(t)\right] \sin \left[w_{c} t+2 \pi k_{f} \int_{-\infty}^{t} m(\tau) \mathrm{d} \tau\right]
\end{aligned}
$$

where $A$ is the amplitude of the normalized $A_{c}$, i.e. $A=1$, then (15) becomes:

$$
\frac{\mathrm{d} s_{\mathrm{FM}}(t)}{\mathrm{d} t}=-\left[w_{c}+2 \pi k_{f} m(t)\right] \sin \left[w_{c} t+2 \pi k_{f} \int_{-\infty}^{t} m(\tau) \mathrm{d} \tau\right]
$$

It can be seen from (16) that the FM signal changed into AM-FM signal after differentiation. Hilbert transformation of the differentiation of the FM signal is:

$$
\begin{aligned}
& \mathrm{H}\left[\frac{\mathrm{d} s_{\mathrm{FM}}(t)}{\mathrm{d} t}\right] \\
& =\left[w_{c}+2 \pi k_{f} m(t)\right] \cdot \exp \left\{\mathrm{j}\left[w_{c} t+2 \pi k_{f} \int_{-\infty}^{t} m(\tau) \mathrm{d} \tau+\pi / 2\right]\right\}
\end{aligned}
$$

Generally, $w_{c}$ is not always greater than $2 \pi k_{f} m(t)$, then (17) turns into:

$$
\begin{gathered}
\mathrm{H}\left[\frac{\mathrm{d} s_{\mathrm{FM}}(t)}{\mathrm{d} t}\right] \\
=\left|w_{c}+2 \pi k_{f} m(t)\right| \cdot \exp \left\{\mathrm{j}\left[w_{c} t+2 \pi k_{f} \int_{-\infty}^{t} m(\tau) \mathrm{d} \tau+\phi(t)\right]\right\} \\
\phi(t)= \begin{cases}\pi / 2, & \text { if } w_{c}+2 \pi k_{f} m(t) \geq 0 \\
3 \pi / 2, & \text { if } w_{c}+2 \pi k_{f} m(t)<0\end{cases}
\end{gathered}
$$

The modulus of (17) is $\left|w_{c}+2 \pi k_{f} m(t)\right|$, and $\phi(t)$ can be obtained by calculating the argument of (18) and relieving the illegibility. Interested readers can refer to papers $[9,10]$ for the concrete algorithms.

When the $\left|w_{c}+2 \pi k_{f} m(t)\right|$ and $\phi(t)$ are obtained, $\theta(t)$ can be expressed as:

$$
\begin{aligned}
\theta(t) & =\left|w_{c}+2 \pi k_{f} m(t)\right| \cdot \exp \{\phi(t)\} \\
& =w_{c}+2 \pi k_{f} m(t)
\end{aligned}
$$

To achieve an expression with the modulation index, we substitute the monotone baseband modulation signal $m(t)=V_{m} \cos \left(2 \pi f_{m} \mathrm{t}\right)$ into (20), subtract $\theta(t)$ by its mean, and make a integration. The following expression can be achieved:

$$
\begin{aligned}
& \int_{0}^{t} 2 \pi k_{f}\{m(\tau)-\operatorname{mean}[m(\tau)]\} \mathrm{d} \tau \\
= & \left.2 \pi k_{f} \cdot \frac{V_{m}}{2 \pi f_{m}} \sin \left(2 \pi f_{m} \tau\right)\right|_{0} ^{t} \\
= & m_{f} \cdot \sin \left(2 \pi f_{m} t\right)
\end{aligned}
$$

where $m_{f}$ is obtained by calculating the amplitude deviation of the sinusoidal signal according to (21). Therefore, the blind estimation of the AM index of FM signal is accomplished.

Using a similar procedure as described in the above section, an integral form for the PM case yields:

$$
\begin{aligned}
\int_{0}^{t} k_{p}\left\{m^{\prime}(\tau)-\operatorname{mean}\left[m^{\prime}(\tau)\right]\right\} \mathrm{d} \tau & =\left.k_{p} V_{m} \cdot \cos \left(2 \pi f_{m} \tau\right)\right|_{0} ^{t} \\
& =m_{p} \cdot\left[\cos \left(2 \pi f_{m} t\right)-1\right]
\end{aligned}
$$

where $m^{\prime}(t)$ is the differentiation of $m(t)$.

Similarly, $m_{p}$ can be obtained by calculating the amplitude deviation of the sinusoidal signal according to (22). Therefore, the AM index of PM signal is also achieved.

By now, a blind AM index estimation algorithm is derived. To be clearer, the main steps of this method are summarized as follows:

Step1. Judging whether the baseband modulation of the input AM signal is monotone or not. If not, estimate the code rate of the signal.

Step2. Normalizing the amplitude of the input signal and differentiating the normalized signal.

Step3. Executing the Hilbert transformation.

Step4. Calculating the modulus and solving $\phi(t)$ by removing phase ambiguity.

Step5. Obtaining $\theta(t)$ by (20) with the estimated modulus and phase.

Step6. Subtracting the mean of $\theta(t)$ and calculating the subsequent integral.

Step7. If the baseband is monotone, the modulation index is estimated according to the amplitude deviation between the adjacent integral values; otherwise, the modulation index is estimated in each code interval respectively.

Step8. Averaging previous estimated values to reduce noise influence on blind estimation of modulation index as little as possible.

\section{Simulations}

Experiments are executed to verify the capabilities of the proposed method. In the experiments, the blind estimation method of the modulation index is realized for the AM signal with baseband modulations, such as monotone, PSK, FSK. Conclusions are drawn according to the simulation results. 


\subsection{Monotone Baseband Case}

The AM signal with monotone baseband is generated. The sampling frequency is $200 \mathrm{MHz}$, the carrier frequency is $70 \mathrm{MHz}$, and $V_{m}=1 \mathrm{~V}$. The following six scenarios are conducted:

1) FM signal with $k_{f}=50 \mathrm{kHz} / \mathrm{V}, f_{m}=40 \mathrm{kHz}$, namely $m_{f}=1.25$;

2) FM signal with $k_{f}=50 \mathrm{kHz} / \mathrm{V}, f_{m}=30 \mathrm{kHz}$, namely $m_{f}=5 / 3$;

3) FM signal with $k_{f}=40 \mathrm{kHz} / \mathrm{V}, f_{m}=30 \mathrm{kHz}$, namely $m_{f}=4 / 3$;

4) $\mathrm{PM}$ signal with $k_{p}=0.4 \mathrm{rad} / \mathrm{V}, f_{m}=40 \mathrm{kHz}$, namely $m_{p}=0.4$;

5) PM signal with $k_{p}=0.8 \mathrm{rad} / \mathrm{V}, f_{m}=40 \mathrm{kHz}$, namely $m_{p}=0.8$;

6) PM signal with $k_{p}=0.8 \mathrm{rad} / \mathrm{V}, f_{m}=20 \mathrm{kHz}$, namely $m_{p}=0.8$.

Normalized deviation and RMSE (Root-Mean-Square Error) of blindly estimated AM index with respect to SNR (Signal-to-Noise Ratio) is shown in Figures 1 and 2.

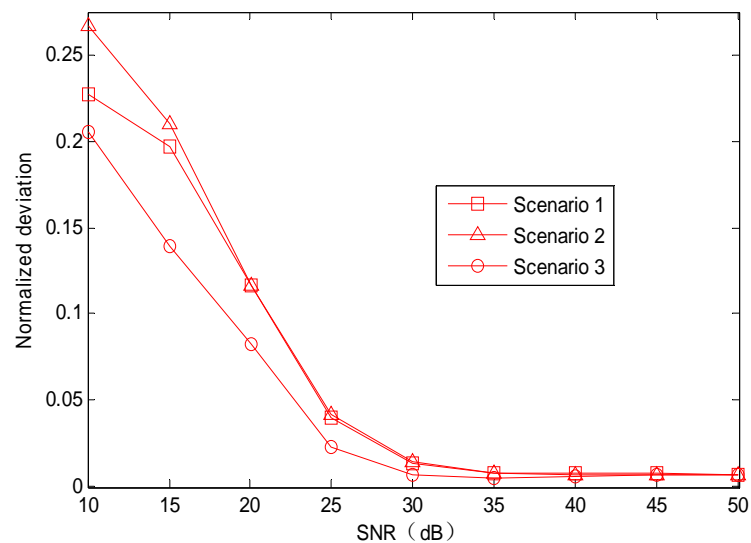

(a)

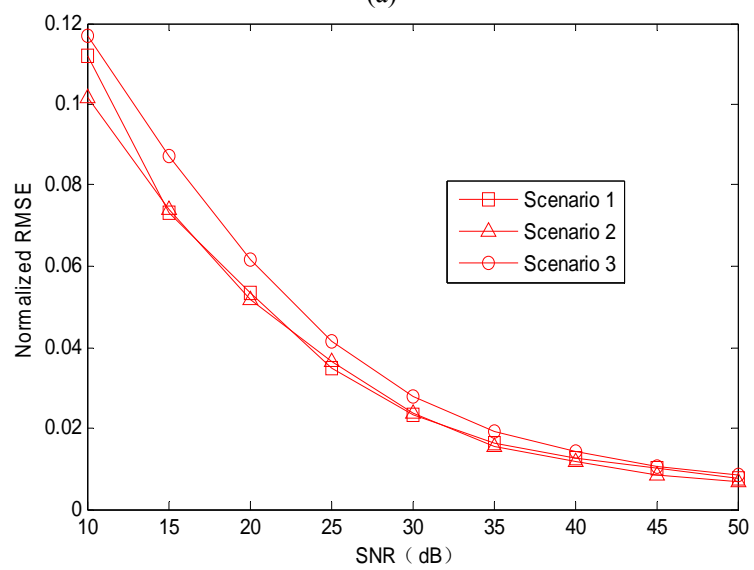

(b)

Figure 1. Blindly estimated result of AM index for FM signal with monotone baseband. (a) Normalized deviation; (b) Normalized RMSE.

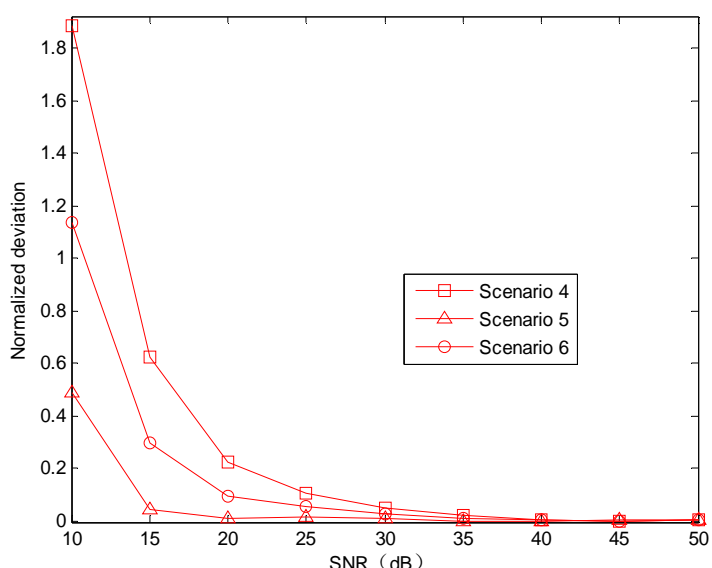

(a)

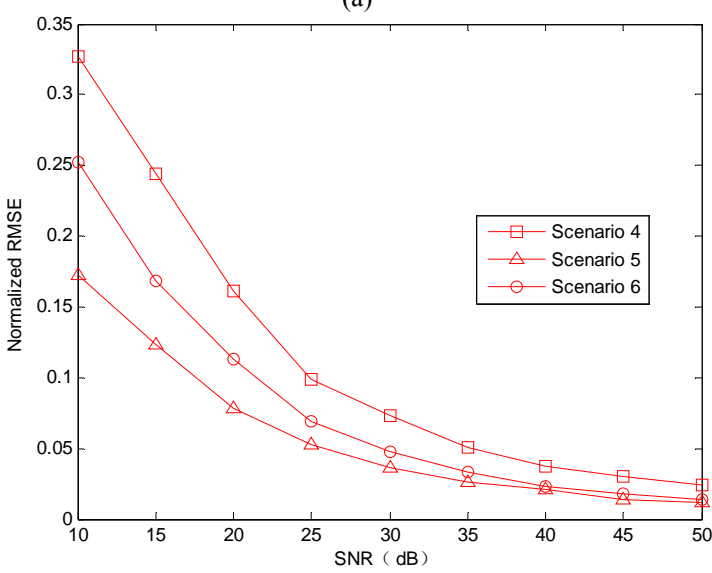

(b)

Figure 2. Blindly estimated result of AM index for PM signal with monotone baseband. (a) Normalized deviation; (b) Normalized RMSE.

The data length used by the algorithm is $1 \mathrm{M}$. The noise is additive white Gaussian noise. Simulation results are averaged from 100 Monte Carlo runs.

\subsection{PSK, FSK Baseband Cases}

The AM signal with BPSK baseband is generated. The sampling frequency is $200 \mathrm{MHz}$, the carrier frequency is $70 \mathrm{MHz}$, and $V_{m}=1 \mathrm{~V}$. The following four simulation scenarios are conducted:

7) FM signal with $k_{f}=50 \mathrm{kHz} / \mathrm{V}, f_{m}=40 \mathrm{kHz}, f_{z}=10$ $\mathrm{kHz}$, namely $m_{f}=1.25$;

8) FM signal with $k_{f}=50 \mathrm{kHz} / \mathrm{V}, f_{m}=30 \mathrm{kHz}, f_{z}=10$ $\mathrm{kHz}$, namely $m_{f}=5 / 3$;

9) PM signal with $k_{p}=0.8 \mathrm{rad} / \mathrm{V}, f_{m}=40 \mathrm{kHz}, f_{z}=10$ $\mathrm{kHz}$, namely $m_{p}=0.8$;

10) PM signal with $k_{p}=0.8 \mathrm{rad} / \mathrm{V}, f_{m}=30 \mathrm{kHz}, f_{z}=10$ kHz, namely $m_{p}=0.8$.

Normalized deviation and RMSE of blindly estimated AM index with respect to SNR is shown in Figure 3. The mean and the variance of estimated error for the code chip 


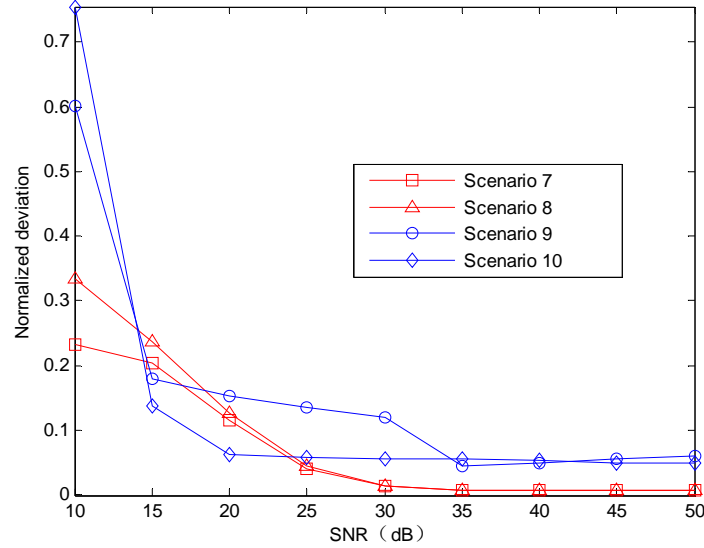

(a)

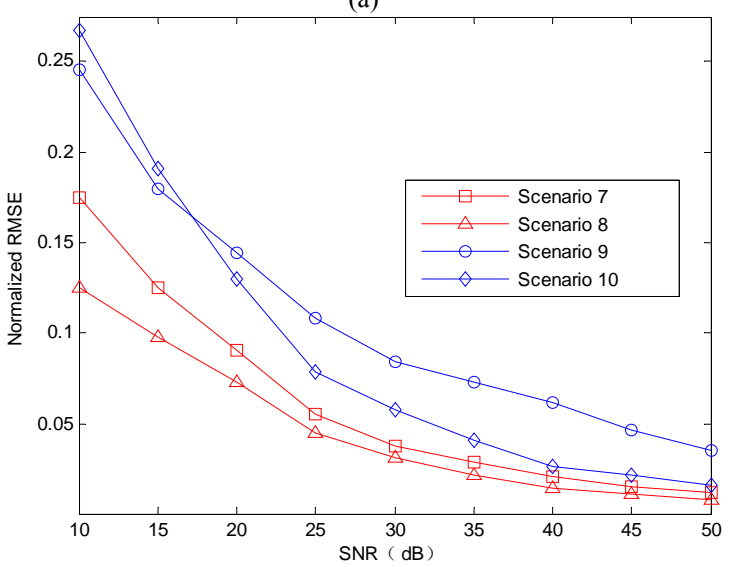

(b)

Figure 3. Blindly estimated result of AM index with BPSK baseband. (a) Normalized deviation; (b) Normalized RMSE.

are $4.9 \times 10^{-5}$ and $3.1 \times 10^{-9}$ respectively [11]. The data length used by the algorithm is $1 \mathrm{M}$. The noise is additive white Gaussian noise. Simulation results are averaged from 100 Monte Carlo runs.

The AM signal with 2 FSK baseband is generated. The sampling frequency is $200 \mathrm{MHz}$, the carrier frequency is $70 \mathrm{MHz}$, and $V_{m}=1 \mathrm{~V}$. The following four simulation scenarios are conducted:

11) FM signal with $k_{f}=50 \mathrm{kHz} / \mathrm{V}, f_{m}=40 \mathrm{kHz}, f_{z}=10$ $\mathrm{kHz}$, frequency interval $20 \mathrm{kHz}$, namely $m_{f}=1.25$;

12) FM signal with $k_{f}=50 \mathrm{kHz} / \mathrm{V}, f_{m}=30 \mathrm{kHz}, f_{z}=10$ $\mathrm{kHz}$, frequency interval $20 \mathrm{kHz}$, namely $m_{f}=5 / 3$;

13) $\mathrm{PM}$ signal with $k_{p}=0.8 \mathrm{rad} / \mathrm{V}, f_{m}=40 \mathrm{kHz}, f_{z}=10$ $\mathrm{kHz}$, frequency interval $20 \mathrm{kHz}$, namely $m_{p}=0.8$;

14) $\mathrm{PM}$ signal with $k_{p}=0.8 \mathrm{rad} / \mathrm{V}, f_{m}=30 \mathrm{kHz}, f_{z}=10$ $\mathrm{kHz}$, frequency interval $20 \mathrm{kHz}$, namely $m_{p}=0.8$.

Normalized deviation and RMSE of blindly estimated AM index with respect to SNR is shown in Figure 4. The data length used by the algorithm is $1 \mathrm{M}$. The noise is additive white Gaussian noise. Simulation results are averaged from 100 Monte Carlo runs.

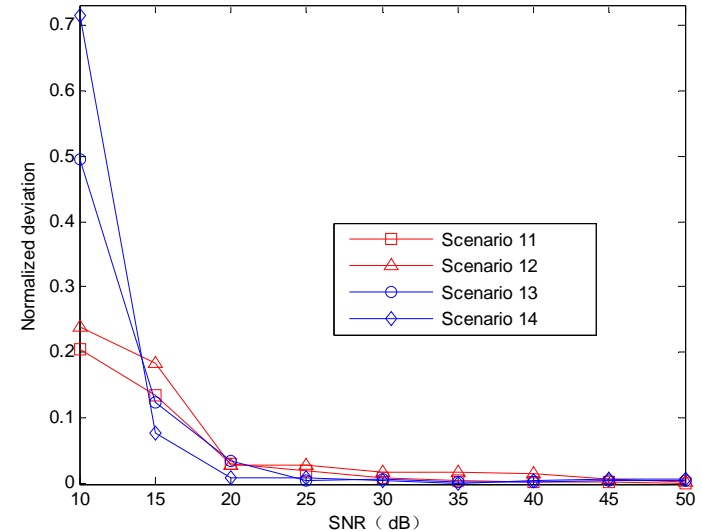

(a)

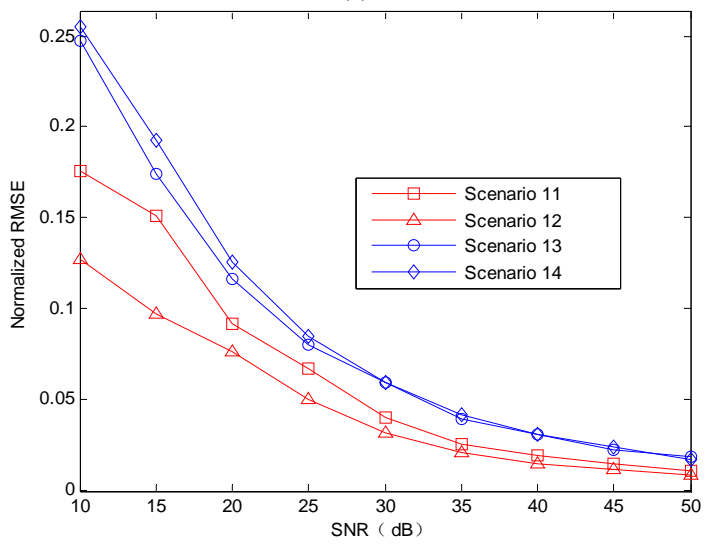

(b)

Figure 4. Blindly estimated result of AM index with 2FSK baseband. (a) Normalized deviation; (b) Normalized RMSE.

\subsection{Analysis of Simulation Results}

According to the above experimental results, conclusions can be drawn as:

1) When $\mathrm{SNR} \geq 20 \mathrm{~dB}$ and the AM signal is modulated by baseband modulations such as monotone, PSK, FSK, the normalized deviation and the RMSE of blindly estimated AM index are smaller than 0.2 and 0.15 respectively. Hence, the algorithm can be used in engineering practice.

2) The normalized deviations and RMSEs of blindly estimated AM index for FM and PM signals are almost the same.

3) Referring to the modulation index range shown in Table 1, high correct identification probabilities can be expected in the recognition of analogue FM and PM signals with the assistance of the AM index estimations when $\mathrm{SNR} \geq 15 \mathrm{~dB}$.

\section{Conclusions}

Based on analyzing of the AM signals in the TF domain, 
this paper establishes a unified mathematical expression for the signal with baseband modulations of monotone, PSK, and FSK. Blind AM index estimation algorithm is further deduced. Moreover, measures to alleviate noise influence are also presented. Simulation results shows that the algorithm is effective, and it can be used in the cases with low SNR. The algorithm can be used in the blind demodulation and the recognition of AM signal for the communication situations without cooperation.

\section{Acknowledgements}

The authors thank the anonymous reviewers for their valuable comments and suggestions which have helped vastly improve the content and presentation of this paper. The authors also acknowledge support from the Innovation Foundation for Outstanding Postgraduates in the National University of Defense Technology of China.

\section{References}

[1] R. W. Jeremy and D. B. Gary, "A Method for Differentiating between Frequency and Phase Modulated Signals," Proceedings of 1999 Information, Decision and Control Conference, Adelaide, February 1999, pp. 489-494.

[2] T. Shintaro and M. Eiichiro, "Automatic Classification of Analogue Modulation Signals by Statistical Parameters," Proceedings of 1999 Military Communications Conference, New Jersey, November 1999, pp. 202-207.

[3] X. W. Zhong, H. Chen and K.-C. Yi, "Automatic Modulation Recognition of TTC Signals of Satellite," Chinese Space Science and Technology, Vol. 23, No. 3, 2003, pp.
57-64.

[4] J. He and Q. Guo, "Automatic Recognition of Modulation Scheme in Satellite TT\&C Channel," Proceedings of the 7th International Conference on Electronic Measurement \& Instrument, Beijing, August 2005.

[5] A. Engin, "Performance Comparison of Wavelet Families for Analog Modulation Classification Using Expert Discrete Wavelet Neural Network System," Expert Systems with Applications, Vol. 33, No. 4, 2007, pp. 23-25.

[6] A. Engin and A. Derya, "Using Combination of Support Vector Machines for Automatic Analog Modulation Recognition," Expert Systems with Applications, Vol. 36, No.1, 2009, pp. 3956-3964.

[7] A. E. EI-Mahdy, "Automatic Modulation Classification of Composite FM/PM Speech Signals in Sensor Arrays over Flat Fading Channel," IET Communications, Vol. 1, No. 2, 2007, pp. 157-164.

[8] X. D. Zhangx and Z. Bao, "Communication Signal Processing," Publishing House of National Defense Industry, Beijing, 2000

[9] Z. T. Huang, Y. Y. Zhou and W. L. Jiang, "The Automatic Analysis of Intra-Pulse Modulation Characteristics Based on the Relatively Non-Ambiguity Phase Restoral,' Journals on Communications, Vol. 24, No. 4, 2003, pp.153-160.

[10] Y. X. Wu, L. D. Ge and Z. Y. Xu, "A Novel Identification Method for Commonly Used Digital Modulations," Acta Electronica Sinica, Vol. 35, No. 4, 2007, pp. 782-785.

[11] Y. F. Zhanx, Z. G. Caox and Z. X. Ma, "Modulation Classification of M-QAM Signals," Journals on Communications, Vol. 25, No. 2, 2004, pp. 68-74. 